\title{
The Impact of Lymphopenia and Dosimetric Parameters on Overall Survival of Esophageal Cancer Patients Treated with Definitive Radiotherapy
}

This article was published in the following Dove Press journal:

Cancer Management and Research

\author{
Ming Liu ${ }^{1,2}$ \\ Xiaoyang $\mathrm{Li}^{3}$ \\ Huaidong Cheng ${ }^{2}$ \\ Yansu Wang ${ }^{4}$ \\ Ye Tian' \\ 'Department of Radiotherapy and \\ Oncology, The Second Affiliated Hospital \\ of Soochow University, Institute of \\ Radiotherapy and Oncology, Soochow \\ University, Suzhou, 215004, People's \\ Republic of China; ${ }^{2}$ Department of \\ Oncology, The Second Affiliated Hospital \\ of Anhui Medical University, Hefei, \\ 23060I, People's Republic of China; \\ ${ }^{3}$ Department of Radiation Oncology, The \\ First Affiliated Hospital of USTS, Division \\ of Life Sciences and Medicine, University \\ of Science and Technology of China, \\ Hefei, 23000I, People's Republic of \\ China; ${ }^{4}$ Department of Radiation \\ Oncology, Shanghai Tenth People's \\ Hospital, Shanghai, 200072, People's \\ Republic of China
}

\begin{abstract}
Purpose: The objectives of the present study are to perform a survival analysis of patients with thoracic esophageal squamous cell carcinoma (ESCC) receiving definitive radiotherapy and to identify prognostic factors from among the hematological and dosimetric factors.

Methods: Cases of thoracic ESCC treated with radical RT between 2014 and 2017 were identified. The impact of clinicopathological factors on overall survival (OS) were analyzed using the Cox proportional hazards model. Absolute lymphocyte counts (ALC) and the neutrophil-to-lymphocyte ratio (NLR $=$ ANC/ALC) were assessed before, during, and after radiotherapy (RT). Cox regression was used to correlate clinical factors with hematologic toxicities, dosimetric parameters and overall survival. Multiple logistic regression analysis was used to identify associations between lymphopenia and dosimetric parameters. With the overall survival status and real time events, the X-tile program was utilized to determine the optimal cut-off value of pretreatment NLR, and ALC nadir.
\end{abstract}

Results: Ninety-nine ESCC patients were enrolled in the present study. They had a median OS of 23 months. The median RT dose was $55.75 \mathrm{~Gy}$ (46-66Gy), and the mean dose ( $\left.\mathrm{D}_{\text {mean }}\right)$ of the thoracic vertebrae (TVB) was $27.04 \pm 9.65 \mathrm{~Gy}$. Based on the multivariate analysis, the V20 of TVB, the pretreatment NLR, and the ALC nadir were associated with significantly worse OS. Concurrent CRT, which entailed increasing the mean TVB dose and V20 of TVB, was linked to a higher probability of lymphopenia risk $(\mathrm{P}<0.05)$. This was ascertained through the multiple logistic regression analysis.

Conclusion: In ESCC patients who received definitive RT, $\mathrm{V}_{20}$ of TVB, pretreatment NLR, and ALC nadir during RT were independent prognostic factors and chemotherapy regimen, mean TVB dose, and $\mathrm{V}_{20}$ of TVB were associated with lymphopenia.

Keywords: radiotherapy, lymphopenia, esophageal cancer, dosimetric parameters, overall survival

\section{Introduction}

Esophageal cancer (EC) is an extremely aggressive type of malignancy with morbidity and mortality ranked sixth and fourth in China, respectively. ${ }^{1}$ In East Asian countries, ESCC is the leading histology subtype and the condition of most patients has progressed to be in the middle or advanced stage. In these cases, and surgery is no longer applicable. ${ }^{2}$ Consequently, CRT or radiation is the definitive non-surgical approach to non-metastatic esophageal cancer. More recently, with the
Department of Radiotherapy and Oncology, The Second Affiliated Hospital of Soochow University, Institute of Radiotherapy and Oncology, Soochow University, Suzhou, 215004, People's Republic of China

Tel +86-5I $2-67783430$

$\mathrm{Fax}+86-512-68284303$

Email dryetian@126.com 
increasing appreciation, immunotherapy, has provided a new paradigm for cancer therapy and has demonstrated significant clinical benefits. ${ }^{3}$ The role of the immune function of patients undergoing radiotherapy has become more of a focal point. ${ }^{4,5}$

Immune and inflammatory responses are the vital processes for tumor progression. ${ }^{6}$ Radiation can suppress host immunity by killing immune cells, in particular cytotoxic T lymphocytes. ${ }^{7}$ During RT, radiation-induced lymphopenia (RIL) is a common hematologic adverse effect because peripheral lymphocytes are known to be the most radiosensitive cells. In the RT procedure, RIL in the tumor microenvironment may promote tumor progression. ALC nadir was proven to be correlated with poor survival in a wide variety of malignancies, such as glioblastoma, cervical, pancreatic, and non-small cell lung cancer (NSCLC).$^{8-11}$ The NLR, an index of generalized inflammatory response, has also been demonstrated as a prognostic factor for patients with multiple cancer types. $^{12,13}$ There is still however, a lack of studies which reveal that immunosuppression interferes with the overall outcomes in ESCC. The purpose of the present study is to identify the effects of hematological toxicity and radiation parameters on the OS of ESCC patients, as well as determine the relationship between lymphopenia and the radiation parameters.

\section{Patients and Methods}

\section{Patient Selection and Data Collection}

We reviewed the medical records of 318 consecutive patients with esophageal cancer who were treated at the Second Affiliated Hospital of Anhui Medical University between February 2014 and November 2017. The specific inclusion criteria were: (1) patients with stage $\mathrm{II}_{-} \mathrm{IV}_{\mathrm{A}}$ esophageal carcinoma (according to the 8th Union for International Cancer Control [UICC] esophageal cancer staging $\left.{ }^{14}\right)$; (2) histological pathologic confirmation that it was limited to squamous cell carcinoma; (3) Eastern Cooperative Oncology Group (ECOG) performance status $\leq 2$; (4) the primary site of esophageal carcinoma was limited to thoracic segments; (5) patients who completed definitive radiotherapy (with or without chemotherapy) of intensity modulated radiation therapy (IMRT) no less than 50Gy without delay; (6) patients that had complete blood counts $(\mathrm{CBC})$ and retrievable dosimetry records (heart, lung, thoracic vertebrae, and whole body doses); and (7) patients without prior chemotherapy, or serious comorbidities that might have affected the lymphocyte count.
The exclusion criteria were: (1) patients who had received surgery; (2) the patients' follow-up was less than one month or unknown; and (3) the necessary and sufficient follow-up data including documented CBC values either before, during, and/or after RT, retrievable and complete DVH information was lacking.

\section{Radiation Treatment and Dosimetric Analysis}

Radiotherapy was conducted using a Varian linear accelerator. All 99 esophageal cancer patients received IMRT. The current practice contoured the gross tumor volume (GTV) according to the chest positioning CT, esophageal barium meal imaging and electronic gastroscopy, which encompassed the primary tumor and positive lymph nodes if present. For the upper thoracic cancer, the clinical tumor volume (CTV) included the involved bilateral supraclavicular and upper mediastinal lymph nodes areas. In the case of middle and lower thoracic cancer, the CTV encompassed the abdominal, middle, and lower mediastinal lymph nodes. CTV was expanded from GTV with 3-4 cm superiorly and inferiorly and $0.8 \mathrm{~cm}$ radially. Planning tumor volume (PTV) was defined as a 0.5 -to 1 -cm expansion from the CTV.

\section{Chemotherapy}

The chemotherapeutic regimen consisted of a weekly intravenous infusion of Cisplatin $\left(25 \mathrm{mg} / \mathrm{m}^{2}\right)$ combined with Fluorouracil $\left(300 \mathrm{mg} / \mathrm{m}^{2}\right.$; continuous infusion) or Paclitaxel $\left(45 \mathrm{mg} / \mathrm{m}^{2}\right)$. It was suspended or the dose was reduced when grade $>2$ hematologic toxicity occurred.

\section{Data Collection}

The following clinicopathologic variables were obtained: of age, gender, location, performance status, stage, use of concurrent or sequential chemotherapy and radiation dosimetry parameters. From CBC data, ALC and absolute neutrophil counts (ANC) were recorded within the two weeks prior to the start of RT. The nadir of ALC was the lowest, appearing within two months after the RT started. The NLR, was calculated by dividing the ANC by the ALC.

The following radiotherapy-related variables were assessed based on the DVH parameters: mean heart dose (MHD), mean lung dose (MLD), mean body dose (MBD), mean vertebral dose (MVD) and V5-20 of TVB from T1T12. The estimated dose of radiation to immune cells (EDRIC) consisting of MHD, MLD, MBD, and the number of fractions as per the model developed by Jin et al, 
confirmed a correlation with OS in NSCLC. ${ }^{15}$ In the present study, we used EDRIC as a composite dosimetric index in the model as follows:

EDRIC $=0.12 * \mathrm{MLD}+0: 08 * \mathrm{MHD}+[0.45+$ $\left.0.35 * 0.85 *(\text { of fractions } / 45)^{1 / 2}\right] * \mathrm{MBD}$

\section{Statistical Analysis}

The primary endpoint was OS, defined as the time between the start date of radiotherapy and the death or final followup (censored) of the patient. The continuous baseline clinicopathological characteristics were analyzed using descriptive statistics, and categorical data were summarized as frequencies and percentages.

The Kaplan-Meier (KM) analysis was performed to estimate survival curves, and the Log rank test was used to compare survival curves. The potential prognostic factors were explored using Cox regression and reported as hazard ratios (HR) and the $95 \%$ confidence intervals. X-tile 3.6.1 software ${ }^{13}$ (Yale University, New Haven, CT, USA) was used to determine the best critical value of the variables within the hematologic index parameters, which included pretreatment NLR and ALC nadir. On this basis, receiver operating characteristics (ROC) curve was used to determine the cut-off points for dosimetric parameters with ALC nadir as state variables during RT. All tests were twosided, and p-values $<0.05$ were considered significant.

All statistical analyses were performed with SPSS version 25.0 (IBM Corp., Armonk, NY, USA) and the survival curves were plotted with GraphPad Prism version 5.01 (GraphPad Software, San Diego, CA, USA).

\section{Results}

Of the 99 patients enrolled in the present study, 79 were male and 20 were female. Their characteristics are listed in Table 1. The median age of all patients included in the present study was 67 years (range, 43-83). For the majority of cases, the cancer was located in the middle segment of the esophagus (55\%), followed by lower esophageal cancer $(25 \%)$, and only 20 cases in the upper segment. There were 55 cases in Stage II, 41 in Stage III and 3 in Stage IVA. The median follow-up of enrolled patients was 24.69 months (range 4-73 months). Most patients received concurrent chemoradiotherapy (59\%), and nine (9\%) of them were treated with sequential chemoradiotherapy. Thirty-two $(32 \%)$ of patients were treated with RT alone due to the toxicity of chemotherapy or self-refusal. The prescribed dose was 46-66 Gy/1.8-2.2 Gy per fraction with a median dose of 55.75 Gy (46-66 Gy), and the $\mathrm{D}_{\text {mean }}$ of TVB was $27.04 \pm 9.65 \mathrm{~Gy}$ (Table 1 ).

\section{Overall Survival and Optimal Cutoff Point} In the entire set of patients, the estimated median OS was 23 months (95\% CI: 18.45-27.56), and three- and fiveyear OS rates were $30 \%$ and $18 \%$, respectively.

To dichotomize the hematologic index parameters, the $\mathrm{X}$-tile software determined that the optimal cut-off value for ALC nadir of OS was $0.3^{*} 10^{9} / \mathrm{L}$ (see in Supplementary Figure 1), and for pretreatment NLR of OS it was 2.0 (see in Supplementary Figure 2), respectively. The $0.3 * 10^{9} / \mathrm{L}$ of ALC nadir was subsequently used as the state viable in ROC analysis which was used to identify the cut-off values of the dosimetric parameters. Cutoffs of $\mathrm{D}_{\text {mean }}$ of TVB was $28.94 \mathrm{~Gy}$, of V5, v10, and V20 of TVB were

Table I Clinicopathological Characteristics

\begin{tabular}{|c|c|}
\hline Characteristics & Median (Range or \%) \\
\hline \multicolumn{2}{|l|}{ Gender } \\
\hline Male & $79(80 \%)$ \\
\hline Female & $20(20 \%)$ \\
\hline Age, years & $67(43-83)$ \\
\hline \multicolumn{2}{|l|}{ ECOG Score } \\
\hline $0-1$ & $69(70 \%)$ \\
\hline 2 & $30(30 \%)$ \\
\hline \multicolumn{2}{|l|}{ Tumor location } \\
\hline Upper & $20(20 \%)$ \\
\hline Middle & $54(55 \%)$ \\
\hline Lower & $25(25 \%)$ \\
\hline \multicolumn{2}{|l|}{ Clinical stage } \\
\hline I & 0 \\
\hline II & $55(56 \%)$ \\
\hline III & $4 \mid(41 \%)$ \\
\hline IVA & $3(3 \%)$ \\
\hline \multicolumn{2}{|l|}{$\mathrm{cN}$ status } \\
\hline No & $42(42 \%)$ \\
\hline $\mathrm{N}+$ & $57(58 \%)$ \\
\hline \multicolumn{2}{|l|}{ Chemotherapy regimen } \\
\hline CRT & $58(59 \%)$ \\
\hline Sequential RT & $9(9 \%)$ \\
\hline RT alone & $32(32 \%)$ \\
\hline Prescribed RT dose (Gy) & $55.75(46-66)$ \\
\hline$<6000$ & 51 (5I\%) \\
\hline$\geq 6000$ & $48(48 \%)$ \\
\hline Dose (Gy)/fraction & $1.8-2.2$ \\
\hline
\end{tabular}



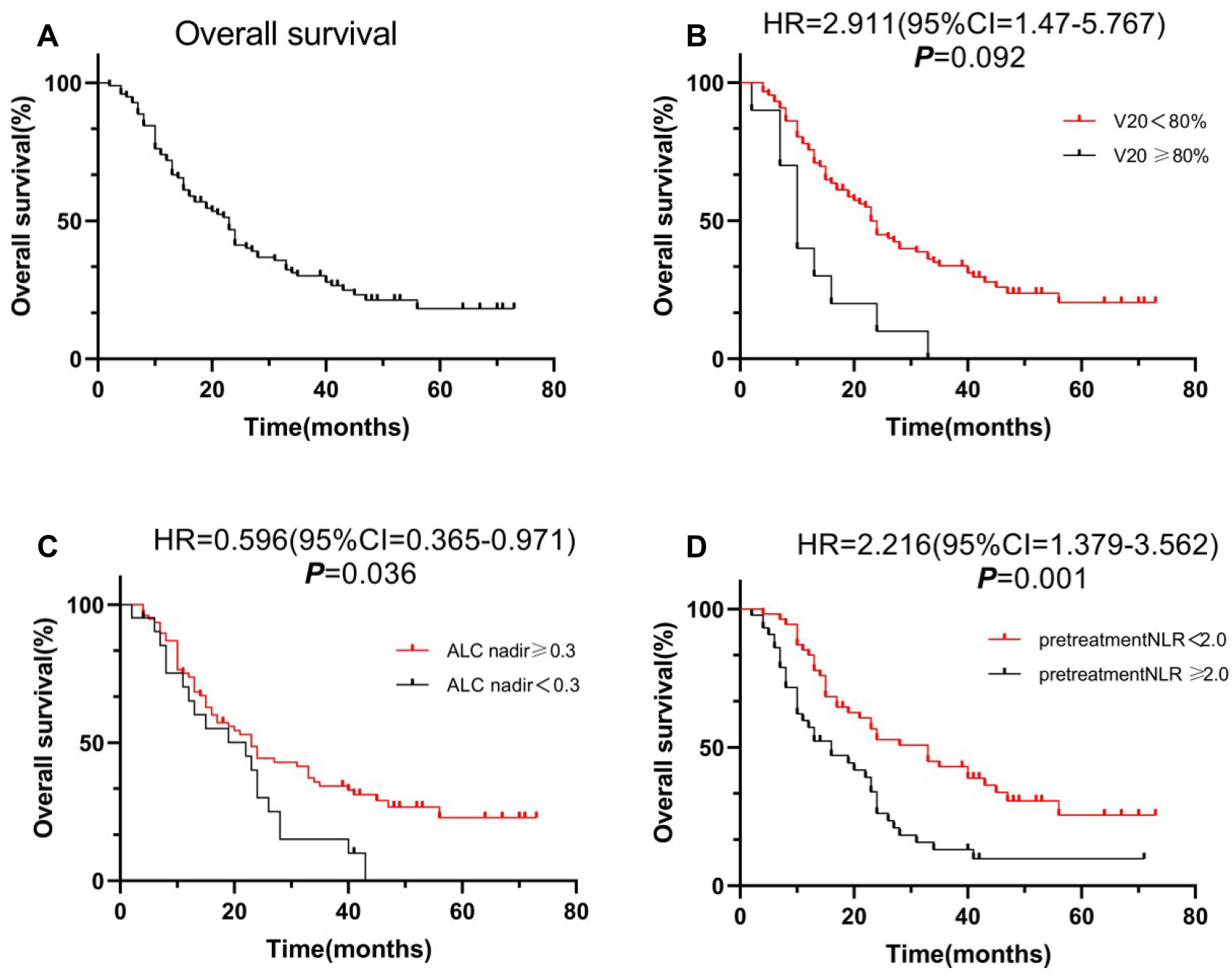

Figure I Kaplan-Meier curves of overall survival for the entire study cohort (A), for patients with V20 of TVB $\geq 80 \%$ versus $<80 \%$ (B), for patients with ALC Nadir $\geq$ $0.3 * 10^{9} / \mathrm{L}$ or $<0.3 * 10^{9} / \mathrm{L}(\mathbf{C})$, and for patients with Pretreatment NLR $\geq 2$ or 2 (D).

$72 \%, 68.1 \%$, and $80 \%$, respectively. The cut-off value of EDRIC was 7.11 Gy. Consequently, the most accurate predictors to avoid ALC nadir below $0.3 * 10^{9} / \mathrm{L}$ were TVB mean doses $\leq 28.94$ Gy, V5 $\leq 72 \%$, V10 $\leq 68.1 \%$, and $\mathrm{V} 20 \leq 80 \%$. EDRIC higher than 7.11 Gy also predicted a lower ALC nadir.

With reference to Figure 1, the Kaplan-Meier curves for OS revealed that patients in the high ALC nadir group, low pretreatment NLR group, and low V20 of TVB group had longer OS. The factors that are correlated with OS are summarized in Table 2. Displayed are the hazard ratios (HRs) and their 95\% confidence intervals (CIs). Based on the univariate analysis, increased $\mathrm{D}_{\text {mean }}$ and $\mathrm{V}_{20}$ of TVB, EDRIC was associated with lower OS. Based on the multivariate analysis, the independent indicators of OS are stage (HR, 2.051; 95\% CI, 1.236-3.405; $P=0.003$ ), pretreatment NLR (HR, 2.062; 95\% CI, 1.278-3.326; $P=0.003)$, ALC nadir (HR, 0.542; 95\% CI, 0.317-0.929; $P=0.026)$, and $\mathrm{V}_{20}$ of TVB (HR, 2.888; 95\% CI, 1.450-5.753; P = 0.003).

\section{Multiple Logistic Regression Analysis of Lymphopenia}

To attain more insights into the impact of the radiation dose on the thoracic vertebrae and lymphopenia, further statistical analyses were performed. The results of a multiple logistic regression analysis is summarized in Table 3. The chemotherapy regimen, EDRIC, $\mathrm{D}_{\text {mean }}$ and $\mathrm{V}_{20}$ of TVB were statistically significantly correlated with the probability of an increase in lymphopenia $(\mathrm{P}<0.05)$.

\section{Discussion}

In the present study, it was discovered that stage, pretreatment NLR, ALC nadir, and $\mathrm{V}_{20}$ of TVB are independent 
Table 2 Cox Regression of Clinical and Dosimetric Variables with Overall Survival

\begin{tabular}{|c|c|c|}
\hline Characteristics & Univariate & Multivariate \\
\hline & HR $(95 \% \mathrm{Cl}), P$ value & HR $(95 \% \mathrm{Cl}), P$ value \\
\hline $\begin{array}{l}\text { Gender } \\
\text { Male vs female }\end{array}$ & NS & NS \\
\hline $\begin{array}{l}\text { Age, years } \\
\leq 65 \text { vs }>65\end{array}$ & NS & NS \\
\hline $\begin{array}{r}\text { ECOG Score } \\
0-1 \text { vs } \geq 2\end{array}$ & NS & NS \\
\hline $\begin{array}{l}\text { Tumor location } \\
\text { Upper vs Middle } \\
\text { Upper vs Lower }\end{array}$ & $\begin{array}{l}\text { NS } \\
\text { NS }\end{array}$ & $\begin{array}{l}\text { NS } \\
\text { NS }\end{array}$ \\
\hline $\begin{array}{l}\text { Chemotherapy regimen } \\
\text { CRT vs Sequential RT } \\
\text { CRT vs RT alone }\end{array}$ & $\begin{array}{l}\text { NS } \\
\text { NS }\end{array}$ & $\begin{array}{l}\text { NS } \\
\text { NS }\end{array}$ \\
\hline $\begin{array}{l}\text { Clinical stage } \\
\text { I II vs III IV }\end{array}$ & $\begin{array}{l}1.893(1.188-3.017) \\
p=0.007\end{array}$ & $\begin{array}{l}2.051(1.236-3.405) \\
p=0.003\end{array}$ \\
\hline $\begin{array}{l}\text { Prescribed RT dose (Gy) } \\
\quad<60 \text { vs } \geq 60\end{array}$ & NS & NS \\
\hline $\begin{array}{l}\text { Pretreatment NLR } \\
\quad<2 \text { vs } \geq 2\end{array}$ & $\begin{array}{l}2.216(1.379-3.562) \\
p=0.003\end{array}$ & $\begin{array}{l}2.062(1.278-3.326) \\
p=0.001\end{array}$ \\
\hline $\begin{array}{l}\text { ALC Nadir }\left(* 10^{9} / \mathrm{L}\right) \\
<0.3 \text { vs } \geq 0.3\end{array}$ & $\begin{array}{l}0.596(0.365-0.971) \\
p=0.026\end{array}$ & $\begin{array}{l}0.542(0.317-0.929) \\
p=0.036\end{array}$ \\
\hline $\begin{array}{l}D_{\text {mean }}(G y) \\
\quad<28.94 \text { vs } \geq 28.94\end{array}$ & $\begin{array}{l}1.623(1.02 \mathrm{I}-2.580) \\
p=0.04 \mathrm{I}\end{array}$ & NS \\
\hline $\begin{array}{l}\text { V5 (\%) } \\
\quad<71.2 \text { vs } \geq 71.2\end{array}$ & NS & NS \\
\hline $\begin{array}{l}\text { VI0 (\%) } \\
<68.9 \text { vs } \geq 68.9\end{array}$ & NS & NS \\
\hline $\begin{array}{l}\text { V20 (\%) } \\
\qquad<80 \text { vs } \geq 80\end{array}$ & $\begin{array}{l}2.911(1.47-5.767) \\
p=0.002\end{array}$ & $\begin{array}{l}2.888(1.450-5.753) \\
p=0.003\end{array}$ \\
\hline $\begin{array}{l}\text { EDRIC (Gy) } \\
\quad<7.11 \text { vs } \geq 7.11\end{array}$ & $\begin{array}{l}1.91(1.089-3.35) \\
p=0.024\end{array}$ & NS \\
\hline
\end{tabular}

predictors of OS for the locally advanced ESCC patients who underwent definitive chemoradiotherapy.

As the marker of inflammation and immunosuppression, pretreatment NLR and ALC nadir during treatment have been used as prognostic factors in various solid tumors, in particular lung cancer. ${ }^{16-18}$

In 2014, Tang et al reported a dataset of 711 NSCLC patients who were treated with definitive radiotherapy and discovered lower lymphocyte nadirs and larger GTVs hence predicting worse outcomes. ${ }^{11}$

Suzuki et $\mathrm{al}^{16}$ on the other hand, reviewed the clinicopathologic and treatment characteristics of 252 patients with ES-SCLC. Using a multivariate analysis, they identified low TLC and high NLR before the treatment as predicting inferior survival. In a recent preclinical study, grade 4 lymphopenia seemed to predict the worst 
Table 3 Multivariate Logistic Regression of the Risk Factors Related to ALC Nadir

\begin{tabular}{|l|l|l|}
\hline \multirow{2}{*}{ Variables } & \multicolumn{2}{|c|}{ Multiple Regression } \\
\cline { 2 - 3 } & \multicolumn{1}{|c|}{ OR (95\% Cl) } & P value \\
\hline $\begin{array}{l}\text { Prescribed RT dose (Gy) } \\
<60 \text { vs } \geq 60\end{array}$ & $1.935(0.659 .5 .683)$ & $p=0.230$ \\
\hline Chemotherapy regimen & & \\
RT alone & Reference & \\
CRT & $4.764(1.555-14.590)$ & $p=0.019$ \\
Sequential RT & $1.026(0.129-8.147)$ & $p=0.981$ \\
\hline Dmean (Gy) & $6.822(1.090-42.706)$ & $p=0.04$ \\
V5 (\%) & $1.333(0.364-4.878)$ & $p=0.664$ \\
VI0 (\%) & $1.447(0.084-2.383)$ & $p=0.346$ \\
V20 (\%) & $1.591(1.336-1.894)$ & $p=0.046$ \\
EDRIC (Gy) & $0.209(0.057-0.762)$ & $p=0.018$ \\
\hline
\end{tabular}

progression-free and OS in an esophageal cancer cohort. ${ }^{19}$ In the present study, we demonstrated that higher pretreatment NLR and lower ALC nadir during definitive RT were significantly associated with worse outcomes.

How does radiation give rise to lymphopenia? Previous preclinical and clinical studies have revealed that radiation interacts with the host immune system by activating innate and adaptive antitumor immune responses. ${ }^{20,21}$ However, the exact mechanism of immunosuppression contributing to radiation-induced lymphopenia remains unresolved. It seems to be to the greater exposure of lymphocyte to the disease sites and larger radiation portals.

The radiation of thoracic malignancies is often close to the heart, such as esophageal, lung and left-sided breast cancers which are encompassed in the radiation portal. Previous studies have highlighted the detrimental effects of lung or heart radiation dose-volumes in lung cancer radiotherapy. This be the case, the higher doses of radiation of the heart, lung and esophagus would result in strong lymphopenia. ${ }^{7,11,22}$ A randomized Phase III clinical trial (RTOG 0617) also revealed the potential for radiation to act as a relevant factor reducing immune function. From a survey of patients who received concurrent CRT of locally advanced NSCLC, heart V5 and V30 were indicated as being predictors of outcomes. From the result of the multivariate analysis, the higher cardiac dose was related to poorer survival. ${ }^{23}$

Another retrospective study of 117 patients who underwent definitive treatment for stage III NSCLC found that the EDRIC was an independent prognosticator of outcomes. ${ }^{15}$ We used the EDRIC model as a reference but did not reach the same conclusions. In our study, EDRIC reflected the statistically significant differences in the univariate analysis only, but not in the multivariate Cox regression. We believe that there are two possible reasons: (1) when the variables of the TVB and EDRIC were simultaneously entered into the Cox regression, an interaction occurred between them, which interfered with the outcome; and/or (2) the relative contribution of EDRIC to the outcome was likely to be relatively small compared with that of the TVB in ESCC patients receiving radical radiotherapy.

In addition to the above viewpoint, the unintentional exposure of the lymph node basins and secondary lymphoid organs to RT like bone marrow, thymus and other potential organs may cause lymphopenia, because these sites and the tumor itself are the key organs induced by a direct hit of lymphocytes by RT. The bone marrow, pelvis, cervical vertebrae, and thoracic vertebrae are the top sites of hematopoiesis because of activation of the proliferating bone marrow. ${ }^{18}$ As a result, RT doses to the pelvic, cervical, and thoracic vertebrae are potential drivers of bone marrow suppression.

Several studies have confirmed that RT dose contributes to bone marrow suppression in the pelvis. ${ }^{24,25}$ Recently, a study of 201 patients with NSCLC and SCLC who received definitive chemoradiation, demonstrated an increased mean TVB dose and V5-V20 of TVB were correlated with higher odds of grade $\geq 3$ hematologic toxicities. ${ }^{15}$ It is important to note that in the present study, only a logistic analysis was performed to explore the correlation of TVB dose with HT3+, and without direct evidence of TVB dose and the overall survival of patients who received CRT.

We assumed in the procedure of chemoradiotherapy that thoracic vertebrae dose correlates with ALC nadir due to immunosuppression. In the present study, the multiple regression analysis confirmed this hypothesis because V20 of TVB $<80 \%$ reduced the risk of ALC nadir $<0.3 * 10^{9} / \mathrm{L}$. The multivariate $\mathrm{COX}$ analysis also indicated that V20 of TVB is an independent predictor for ESCC patients in our cohort. To the best of my knowledge, this is the first research into vertebral RT dose and the prognosis of esophageal cancer.

The present study did have several limitations. First, the retrospective study enrolled patients from a single center in China, which created the risk of bias in selection as well as information. Second, our patient cohort was 
comprised of only 99 patients. This posted a challenge as we applied several radiation parameters including the $\mathrm{D}_{\text {mean }}$ and V5-50 of the heart, and $\mathrm{D}_{\text {mean }}$ and V5-30 of the lungs but did not enter them into the Cox regression due to the small patient cohort. Finally, it was noted that identifying the cut-off values of the categorical data by using X-tile and ROC curve analysis may not provide the most accurate results.

It is on this basis that a larger, multi-institutional study is necessary to verify our results.

\section{Conclusion}

In conclusion, our study demonstrated that $\mathrm{D}_{\text {mean }}$ and V20 of TVB might be clinically useful in predicting severe lymphopenia in patients with locally advanced ECSS receiving definitive radiotherapy. Increased V20 of TVB, as well as pretreatment NLR and decreased ALC nadir were associated with poorer clinical outcomes. $A D_{\text {mean }}$ of TVB below 28.49Gy and TVB V20 $\leq 80 \%$ are correlated with lower ALC nadir. Clinical outcomes can be improved by optimizing prescriptions or implementing treatment planning approaches that minimize the mean dose and V20 of the TVB.

\section{Abbreviations}

ESCC, Esophageal squamous cell carcinoma; OS, Overall survival; ALC, Absolute lymphocyte counts; ANC, Absolute neutrophil counts; NLR, Neutrophil-tolymphocyte ratio; RT, Radiotherapy; ROC, Receiver operating characteristics; $\mathrm{D}_{\text {mean, Mean does; TVB, }}$ Thoracic vertebrae; CRT, Chemoradiation therapy; EC, Esophageal cancer; RIL, Radiation-induced lymphopenia; NSCLC, Non-small cell lung cancer; UICC, Union for International Cancer Control; ECOG, Eastern Cooperative Oncology Group; IMRT, Intensity modulated radiation therapy; $\mathrm{CBC}$, Complete blood counts; DVH, Dose-volume histogram; GTV, Gross tumor volume; CTV, Clinical tumor volume; PTV, Planning tumor volume; MHD, Mean heart dose; MLD, Mean lung dose; MBD, Mean body dose; MVD, Mean vertebral dose; EDRIC, Estimated dose of radiation to immune cells; HR, Hazard ratios.

\section{Data Sharing Statement}

The datasets used during the current study are available from the corresponding author on reasonable request.

\section{Ethics Approval and Consent to Participate}

This study was approved by Ethics Committee of Anhui Medical University (approval No. 20,180,033) and this study was conducted in accordance with the Declaration of Helsinki. Informed consent was obtained from all individual participants included in the study.

\section{Acknowledgments}

We would like to thank the participants who made this study possible. Thanks for the physicists from the Department of Oncology of the Second Affiliated Hospital of Anhui Medical University, for their helping of dosimetric parameters' collection.

\section{Funding}

The present study was supported by the National Natural Science Foundation of China (No. 81872504), the Fundamental Research Funds for the Central Universities (WK9110000177), and Jiangsu Medical Innovation Team (CXDT-37).

\section{Disclosure}

All authors declare that they have no conflicts of interest.

\section{References}

1. Chen W, Zheng R, Baade PD, et al. Cancer statistics in China, 2015. CA Cancer J Clin. 2016;66(2):115-132. doi:10.3322/caac.21338

2. Gao YB, Chen ZL, Li JG, et al. Genetic landscape of esophageal squamous cell carcinoma. Nat Genet. 2014;46(10):1097-1102. doi:10.1038/ng.3076

3. Marrone KA, Naidoo J, Brahmer JR. Immunotherapy for lung cancer: no longer an abstract concept. Semin Respir Crit Care Med. 2016;37 (5):771-782. doi: $10.1055 / \mathrm{s}-0036-1592298$

4. Liang H, Deng L, Chmura S, et al. Radiation-induced equilibrium is a balance between tumor cell proliferation and T cell-mediated killing. J Immunol. 2013;190(11):5874-5881. doi:10.4049/jimmunol.1202612

5. Weichselbaum RR, Liang H, Deng L, Fu YX. Radiotherapy and immunotherapy: a beneficial liaison? Nat Rev Clin Oncol. 2017;14 (6):365-379.

6. Schreiber RD, Old LJ, Smyth MJ. Cancer immunoediting: integrating immunity's roles in cancer suppression and promotion. Science. 2011;331(6024):1565-1570. doi:10.1126/science. 1203486

7. Contreras JA, Lin AJ, Weiner A, et al. Cardiac dose is associated with immunosuppression and poor survival in locally advanced non-small cell lung cancer. Radiother Oncol. 2018;128(3):498-504. doi:10.1016/ j.radonc.2018.05.017

8. Mendez JS, Govindan A, Leong J, Gao F, Huang J, Campian JL. Association between treatment-related lymphopenia and overall survival in elderly patients with newly diagnosed glioblastoma. $J$ Neurooncol. 2016;127(2):329-335. doi:10.1007/s11060-015-2037-1

9. Wu ES, Oduyebo T, Cobb LP, et al. Lymphopenia and its association with survival in patients with locally advanced cervical cancer. Gynecol Oncol. 2016;140(1):76-82. doi:10.1016/j.ygyno.2015.1 1.013 
10. Wild AT, Ye X, Ellsworth SG, et al. The association between chemoradiation-related lymphopenia and clinical outcomes in patients with locally advanced pancreatic adenocarcinoma. Am J Clin Oncol. 2015;38(3):259-265. doi:10.1097/COC.0b013e3182940ff9

11. Tang C, Liao Z, Gomez D, et al. Lymphopenia association with gross tumor volume and lung V5 and its effects on non-small cell lung cancer patient outcomes. Int J Radiat Oncol Biol Phys. 2014;89 (5):1084-1091. doi:10.1016/j.ijrobp.2014.04.025

12. Templeton AJ, Ace O, McNamara MG, et al. Prognostic role of platelet to lymphocyte ratio in solid tumors: a systematic review and meta-analysis. Cancer Epidemiol Biomarkers Prev. 2014;23 (7):1204-1212. doi:10.1158/1055-9965.EPI-14-0146

13. Guo JC, Lin CC, Lin CY, et al. Neutrophil-to-lymphocyte ratio and use of antibiotics associated with prognosis in esophageal squamous cell carcinoma patients receiving immune checkpoint inhibitors. Anticancer Res. 2019;39(10):5675-5682. doi:10.21873/anticanres.13765

14. Rice TW, Ishwaran H, Ferguson MK, Blackstone EH, Goldstraw P. Cancer of the esophagus and esophagogastric junction: an eighth edition staging primer. $J$ Thorac Oncol. 2017;12(1):36-42. doi:10.1016/j.jtho.2016.10.016

15. Ladbury CJ, Rusthoven CG, Camidge DR, Kavanagh BD, Nath SK. Impact of radiation dose to the host immune system on tumor control and survival for stage III non-small cell lung cancer treated with definitive radiation therapy. Int J Radiat Oncol Biol Phys. 2019;105 (2):346-355. doi:10.1016/j.ijrobp.2019.05.064

16. Suzuki R, Lin SH, Wei X, et al. Prognostic significance of pretreatment total lymphocyte count and neutrophil-to-lymphocyte ratio in extensive-stage small-cell lung cancer. Radiother Oncol. 2018;126 (3):499-505. doi:10.1016/j.radonc.2017.12.030

17. Joseph N, McWilliam A, Kennedy J, et al. Post-treatment lymphocytopaenia, integral body dose and overall survival in lung cancer patients treated with radical radiotherapy. Radiother Oncol. 2019;135:115-119. doi:10.1016/j.radonc.2019.03.008

18. Venkatesulu BP, Mallick S, Lin SH, Krishnan S. A systematic review of the influence of radiation-induced lymphopenia on survival outcomes in solid tumors. Crit Rev Oncol Hematol. 2018;123:42-51. doi:10.1016/j.critrevonc.2018.01.003
19. van Rossum PSN, Deng W, Routman DM, et al. Prediction of severe lymphopenia during chemoradiation therapy for esophageal cancer: development and validation of a pretreatment nomogram. Pract Radiat Oncol. 2020;10(1):e16-e26. doi:10.1016/j.prro.2019.07.010

20. Grass GD, Krishna N, Kim S. The immune mechanisms of abscopal effect in radiation therapy. Curr Probl Cancer. 2016;40(1):10-24. doi:10.1016/j.currproblcancer.2015.10.003

21. Bernstein MB, Krishnan S, Hodge JW, Chang JY. Immunotherapy and stereotactic ablative radiotherapy (ISABR): a curative approach? Nat Rev Clin Oncol. 2016;13(8):516-524. doi:10.1038/nrclinonc.2016.30

22. Davuluri R, Jiang W, Fang P, et al. Lymphocyte nadir and esophageal cancer survival outcomes after chemoradiation therapy. Int $J$ Radiat Oncol Biol Phys. 2017;99(1):128-135. doi:10.1016/j.ijrobp.2017.05.037

23. Bradley JD, Paulus R, Komaki R, et al. Standard-dose versus high-dose conformal radiotherapy with concurrent and consolidation carboplatin plus paclitaxel with or without cetuximab for patients with stage IIIA or IIIB non-small-cell lung cancer (RTOG 0617): a randomised, two-by-two factorial Phase 3 study. Lancet Oncol. 2015;16(2):187-199. doi:10.1016/S1470-2045(14)71207-0

24. Albuquerque K, Giangreco D, Morrison C, et al. Radiation-related predictors of hematologic toxicity after concurrent chemoradiation for cervical cancer and implications for bone marrow-sparing pelvic IMRT. Int $J$ Radiat Oncol Biol Phys. 2011;79(4):1043-1047. doi:10.1016/j.ijrobp.2009.12.025

25. Bazan JG, Luxton G, Mok EC, Koong AC, Chang DT. Normal tissue complication probability modeling of acute hematologic toxicity in patients treated with intensity-modulated radiation therapy for squamous cell carcinoma of the anal canal. Int J Radiat Oncol Biol Phys. 2012;84(3):700-706. doi:10.1016/j.ijrobp.2011.12.072

\section{Publish your work in this journal}

Cancer Management and Research is an international, peer-reviewed open access journal focusing on cancer research and the optimal use of preventative and integrated treatment interventions to achieve improved outcomes, enhanced survival and quality of life for the cancer patient.
The manuscript management system is completely online and includes a very quick and fair peer-review system, which is all easy to use. Visit http://www.dovepress.com/testimonials.php to read real quotes from published authors. 